\title{
Stand structure and height-diameter relationship of a degraded Populus euphratica forest in the lower reaches of the Tarim River, Northwest China
}

\author{
AISHAN Tayierjiang ${ }^{1,2}$, HALIK Ümüt ${ }^{1,2^{*}}$, Florian BETZ ${ }^{2}$, TIYIP Tashpolat $^{1}$, DING Jianli ${ }^{1}$, NUERMAIMAITI \\ Yiliyasijiang $^{1}$ \\ ${ }^{1}$ Key Laboratory of Oasis Ecology, College of Resource \& Environmental Sciences, Xinjiang University, Urumqi 830046, China; \\ ${ }^{2}$ Faculty of Geography and Mathematics, Catholic University of Eichstaett-Ingolstadt, Eichstaett 85071, Germany
}

\begin{abstract}
Understanding stand structure and height-diameter relationship of trees provides very useful information to establish appropriate countermeasures for sustainable management of endangered forests. Populus euphratica, a dominant tree species along the Tarim River watershed, plays an irreplaceable role in the sustainable development of regional ecology, economy and society. However, as the result of climate changes and human activities, the natural riparian ecosystems within the whole river basin were degraded enormously, particularly in the lower reaches of the river where about $320 \mathrm{~km}$ of the riparian forests were either highly degraded or dead. In this study, we presented one of the main criteria for the assessment of vitality of $P$. euphratica forests by estimating the defoliation level, and analyzed forest structure and determined the height-diameter (height means the height of a tree and diameter means the diameter at breast height ( $\mathrm{DBH})$ of a tree) relationship of trees in different vitality classes (i.e. healthy, good, medium, senesced, dying, dead and fallen). Trees classified as healthy and good accounted for approximately $40 \%$ of all sample trees, while slightly and highly degraded trees took up nearly $60 \%$ of total sample trees. The values of TH (tree height) and DBH ranged from 0-19 m and 0-125 cm, respectively. Trees more than $15 \mathrm{~m}$ in TH and $60 \mathrm{~cm}$ in DBH appeared sporadically. Trees in different vitality classes had different distribution patterns. Healthy trees were mainly composed more of relatively younger trees than of degraded tress. The height-diameter relationships differed greatly among tress in different vitality classes, with the coefficients ranging from 0.1653 to 0.6942 . Correlation coefficients of TH and DBH in healthy and good trees were higher than those in trees of other vitality classes. The correlation between TH and DBH decreased with the decline of tree vitality. Our results suggested that it might be able to differentiate degraded $P$. euphratica trees from healthy trees by determining the height-diameter correlation coefficient, and the coefficient would be a new parameter for detecting degradation and assessing sustainable management of floodplain forests in arid regions. In addition, tree vitality should be taken into account to make an accurate height-diameter model for tree height prediction.
\end{abstract}

Keywords: stand structure; height-diameter relationship; Populus euphratica; tree vitality; Tarim River

Citation: AISHAN Tayierjiang, HALIK Ümüt, Florian BETZ, TIYIP Tashpolat, DING Jianli, NUERMAIMAITI Yiliyasijiang. 2015. Stand structure and height-diameter relationship of a degraded Populus euphratica forest in the lower reaches of the Tarim River, Northwest China. Journal of Arid Land , 7(4): 544-554. doi: 10.1007/s40333-015-0046-8

Populus euphratica Oliv. is the dominant tree species in the riparian forest ecosystems along the arid inland rivers of China. More than $90 \%$ of this existing tree species in China are distributed in the Tarim River Basin of Xinjiang (Wang et al., 1996; Huang, 2002).
In the easternmost part of the Tarim River, P. euphratica forests form so called "Green Corridor" to prevent the Taklimakan Desert and Kumtag Desert from merging together (Song et al., 2000). Meantime, as the natural barrier for all economic activities, they

*Corresponding author: HALIK Ümüt (E-mail: uemuet.halik@ku.de)

Received 2014-12-03; revised 2015-01-29; accepted 2015-02-06

(C) Xinjiang Institute of Ecology and Geography, Chinese Academy of Sciences, Science Press and Springer-Verlag Berlin Heidelberg 2015 
also provide a wide range of ecosystem services such as resisting sandstorms, moderating desertification, regulating oasis climate, fertilizing forest soil, protecting biodiversity and maintaining ecosystem balance (Aishan et al., 2013; Cyffka et al., 2013). Most importantly, the forests act as an effective shelterbelt that protects the National Highway No. 218 running through the "Green Corridor" from sand drift (Kuba et al., 2013). For these reasons, P. euphratica has been regarded as one of the key target species to be protected.

However, due to climate change and excessive utilization of water resources over the past 50 years, $P$. euphratica forests along the lower reaches of the Tarim River have been severely damaged which, at the same time, exacerbated desertification process and led to massive degradation of ecological environment in this region (Aishan et al., 2013; Ling et al., 2014; Yang and Yi, 2014; Ye et al., 2014). In order to restore the watershed ecosystem in the lower reaches of the Tarim River, Chinese government has invested more than 10 billion RMB to implement the Ecological Water Conveyance Program (EWCP) since 2000. As the increasing of water amount and frequency, groundwater level is rising and riparian forests are showing different degrees of recovery (Halik et al., 2006, 2009; Aishan et al., 2013; Chen et al., 2013; Hao and Li, 2014; Peng et al., 2014). To quantitatively and qualitatively assess the effectiveness of EWCP, many scholars have conducted great deal of researches from different prospectives (Song et al., 2000; Chen et al., 2006; Halik et al., 2006; Xu et al., 2007; Thevs et al., 2008; Chen et al., 2014; Liu et al., 2014; Aishan et al., 2015). Studies related to the stand structure of $P$. euphratica and relationships between ecological parameters of this tree species were carried out at home and abroad (Wang et al., 2006; Westermann et al., 2008; Yukako et al., 2008; Thevs et al., 2012).

Until recently, however, the research regarding stand structure and relationship between tree height (TH) and diameter at breast height (DBH) of degraded P. euphratica forests in the lower reaches of the Tarim River has not been reported. The growth of tree is not only determined by the physiological and ecological processes of tree, but also by site quality (Wang et al., 2006). Height-diameter relationship of tree is closely associated with habitat conditions and quality of tree itself. Under normal situation, there is a strong biological height-diameter relationship of tree ( $\mathrm{Li}$ and $\mathrm{Fa}$, 2011). Height-diameter correlation of tree more or less reflects the ecological conditions of forest tree and can be a major indicator for evaluating the quality of riparian forest ecosystem. In order to understand the stand structure and the effects of forests with different degradation degrees on the relationships between ecological indicators of $P$. euphratica forests under current restoration practices, we estimated tree vitality of P. euphratica forests at Arghan section along the lower reaches of the Tarim River, and analyzed the distributions of TH and DBH and the height-diameter relationships of $P$. euphratica forests in different vitalities. Our results can be used to further assess the effectivenesses of ecological water conveyance, and conservation and sustainable management of remaining riparian forests in future practices.

\section{Study area}

The study area is located at Arghan transect $\left(40^{\circ} 08^{\prime} 50^{\prime \prime} \mathrm{N}, 88^{\circ} 21^{\prime} 28^{\prime \prime} \mathrm{E}\right)$ between Daxihaizi Reservoir and Taitema Lake in the lower reaches of the Tarim River, Xinjiang Uygur autonomous region, Northwest China (Fig. 1). This area is characterized by an extremely arid climate, with an average annual precipitation of $<50 \mathrm{~mm}$ (Song et al., 2000) and a potential annual evaporation of 2,500-3,000 mm (Chen et al., 2006; Aishan et al., 2013). Vegetation is mainly distributed along the two sides of the Tarim River and composes the typical riparian ecosystem, which comprises trees, shrubs and herbs. The main trees are Populus euphratica Oliv. and Elaeagnus angustifolia L.; and main shrubs are Tamarix ramosissima Ledeb., Tamarix hispida Willd., Tamarix elongate Ledeb., Lycium ruthenicum Murr., Halimodendron halodendron (Pall.) Voss., Halostachys caspica (M. B.) C. A. Mey., Poacynum hendersonii (Hook. F.) Woodson., Alhagi sparsifolia (B. Keller et Shap.) Shap., Glyzrrhiza inflate Bat., Karelinia caspica (Pall.) Less., Inula salsoloides (Turcs.) Ostrnf. and Hexinia polydichotoma (Osten.) H. L. Yang (Halik et al., 2006; Thevs et al., 2012). Annual average groundwater depth varies from 1 to $11 \mathrm{~m}$ (Aishan et al., 2015). Vegetation is highly dependent on the favorable groundwater depth recharged by water diversion project. 


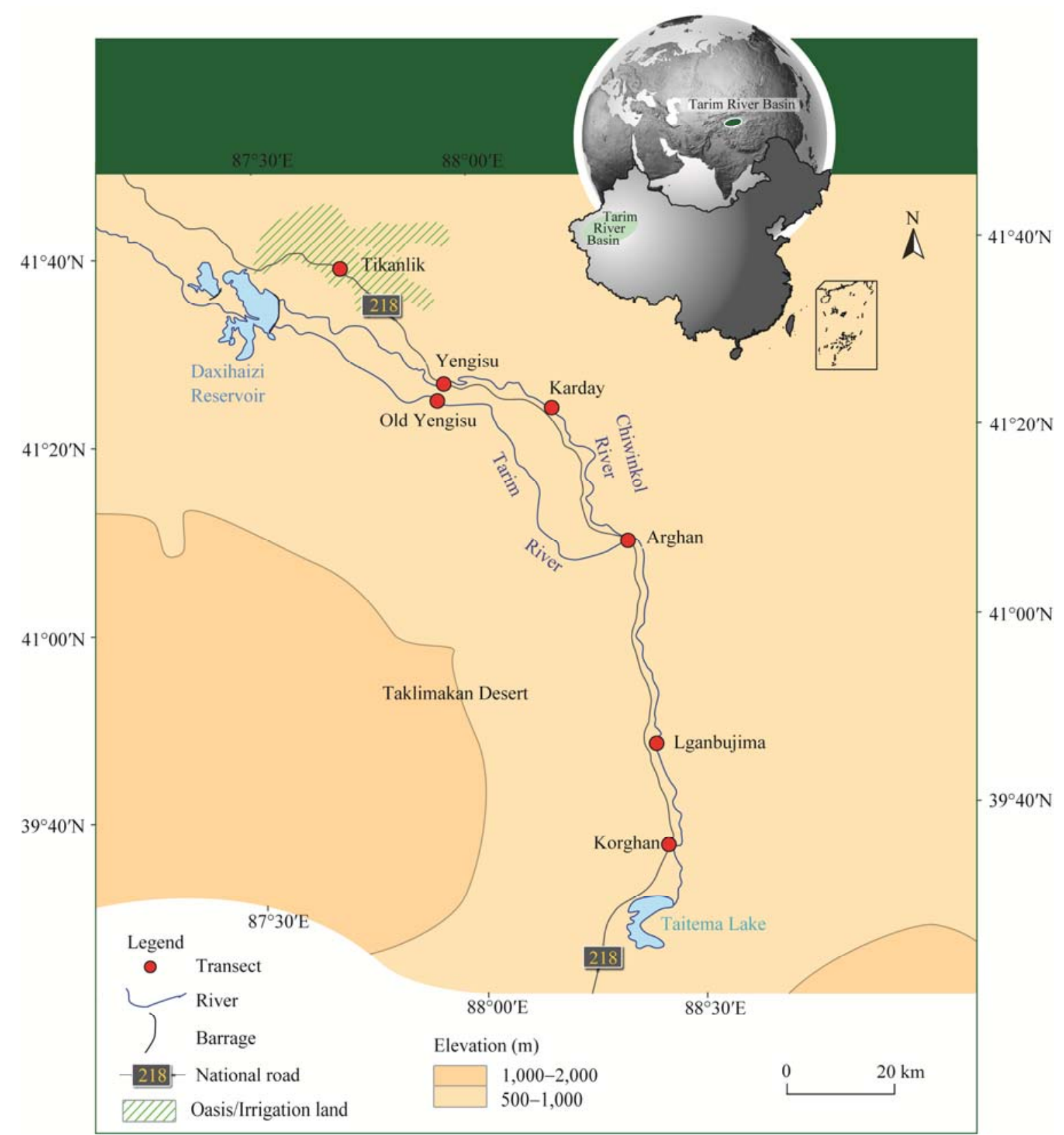

Fig. 1 Geographical location of the study area (Arghan transect) in the lower reaches of the Tarim River, Xinjiang of China

\section{Materials and methods}

\subsection{Monitoring plots and measurements}

By using groundwater data from six timely groundwater monitoring wells established by the Tarim River Basin Water Administration Bureau, we explored the effects of ecological water conveyance on recoveries of degraded trees and fluctuations of groundwater levels. We designed 100 long-term sample plots with each area of $100 \mathrm{~m} \times 100 \mathrm{~m}$ at Arghan transect. These long-term sample plots had a total area of $100 \mathrm{hm}^{2}$ in which more than 4,500 P. euphratica trees were labeled. From May to September 2011, we measured plant species, coverage and canopy density, individual tree parameters $(\mathrm{TH}, \mathrm{DBH}$, crown diameter and under branch height) and visual assessment indicators (vitality class, crown type, leaf loss and shoot branches) within these plots. Extensive terrestrial investigation will be continuously conducted at an interval of 5-year (changes in ecological parameters of trees are small because of extremely harsh environment). 


\subsection{Tree vitality and classification}

Tree vitality, an integrated concept associated with the physiology, ecology and morphology of forests, refers to the growth status and variations of forests (including crown, leaves, stems and branches) as well as extension of canopy (Aishan et al., 2015). It can be a key indicator to assess the overall situations of forests, e.g. health conditions, integrity and resilience. Based on the visual classification criteria (Lam et al., 2010; Aishan et al., 2015) for vitality of $P$. euphratica trees (Table 1; Fig. 2), we divided trees in the study area into seven classes: healthy tree (V0), good tree (V1), medium tree (V2), senesced tree (V3), dying tree (V4), dead tree (V5) and fallen tree (V6). We did not record detailed ecological parameters of fallen trees except for the explanation of overall status during the field work. Therefore, further analysis for fallen trees was not presented in this study. The distribution of $P$. euphratica trees in different vitalities in this study is shown in Fig. 3.

A large proportion of $P$. euphratica trees in healthy and good classes (1,761 trees) were found in our study area (Table 2). Slightly and highly degraded trees (e.g. medium, senesced, dying and dead trees; 2,556 trees)

Table 1 Classification criteria for the vitality of Populus euphratica trees

\begin{tabular}{cccl}
\hline Code & Vitality class & Leaf loss $(\%)$ & Overall status and crown features \\
\hline V0 & Healthy & $\leq 10$ & Vital tree (almost) without signs of damage, healthy full primary crown, usually holds dark green leaves \\
V1 & Good & $11-25$ & Crown slightly damaged, but still in good condition, less than $25 \%$ loss of crown \\
V2 & Medium & $26-50$ & Crown moderately damaged, some primary and secondary crown present, crown loss in half \\
V3 & Senesced & $51-75$ & Crown heavily damaged, tendency to deterioration (e.g. extant dried leaves), crown loss under 75\% \\
V4 & Dying & $76-99$ & $\begin{array}{l}\text { Primary crown very severely damaged, missing or secondary crown is also damaged, evidence of } \\
\text { residual vitality (e.g. single green leaves), tree almost strays }\end{array}$ \\
V5 & Dead & 100 & Standing dead wood, no evidence of (residual) vitality \\
V6 & Fallen & 100 & Lying dead wood, stumps \\
\hline
\end{tabular}

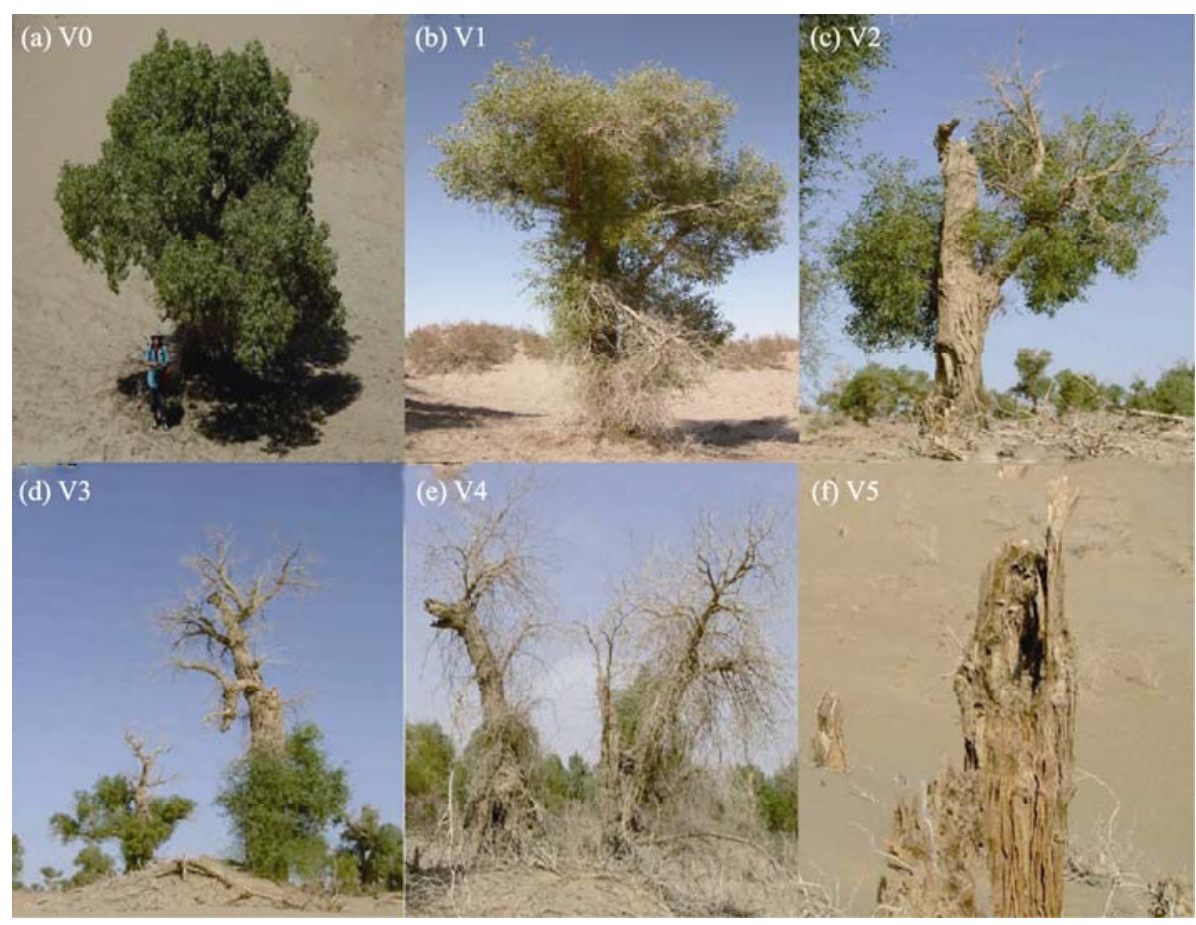

Fig. 2 Photos of Populus euphratica in different vitality classes. V0, healthy tree; V1, good tree; V2, medium tree; V3, senesced tree; V4, dying tree; V5, dead tree. 


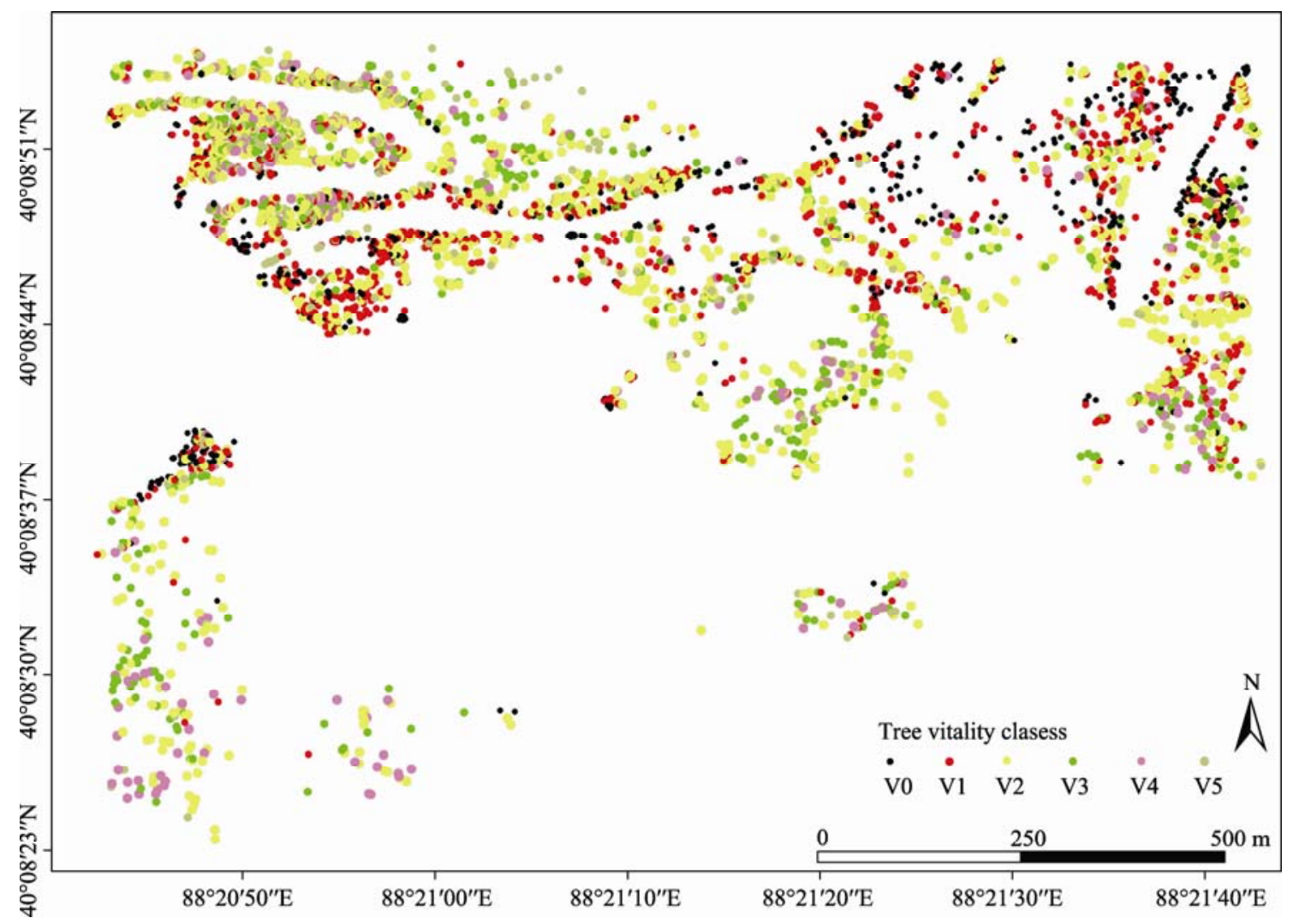

Fig. 3 Distribution of $P$. euphratica trees in different vitality classes in the study area

occupied nearly $60 \%$ of the total sample trees. An interesting point was that mean values of $\mathrm{DBH}$ and $\mathrm{TH}$ increased as the decreasing of tree vitality except dead trees. This indicated that healthy trees are mainly composed by the relatively young trees. It should also be clarified that most of the dead trees are distributed far away from the river, where they could not be supported by sufficient water for a long time. Therefore, regrowth of dead trees are highly limited in this harsh environment.

\subsection{Data processing}

Both terrestrial vegetation data and QuickBird high resolution satellite image were used in this study. A total of 4,500 P. euphratica trees within 100 long-term sample plots were monitored individually by extensive terrestrial investigations in combination with high resolution QuickBird satellite image. Before the establishment of these long-term sample plots, mean or low resolution satellite images $(\mathrm{TM} / \mathrm{ETM}+$, SPOT and

Table 2 Summary statistics of measured parameters (DBH and TH) of $P$. euphratica trees in different vitalities

\begin{tabular}{|c|c|c|c|c|c|c|c|c|c|}
\hline \multirow{2}{*}{ Vitality class } & \multirow{2}{*}{ Number of trees } & \multicolumn{4}{|c|}{$\mathrm{DBH}(\mathrm{cm})$} & \multicolumn{4}{|c|}{ TH (m) } \\
\hline & & Min. & Mean & Max. & SD & Min. & Mean & Max. & SD \\
\hline V0 & 692 & 3.80 & 15.90 & 85.00 & 14.67 & 1.20 & 5.32 & 15.70 & 2.79 \\
\hline V1 & 1,069 & 4.20 & 25.31 & 80.00 & 14.67 & 1.20 & 6.97 & 19.00 & 2.79 \\
\hline V2 & 1,342 & 9.22 & 26.83 & 110.50 & 12.31 & 1.00 & 6.69 & 18.40 & 2.63 \\
\hline V3 & 617 & 14.20 & 30.22 & 119.00 & 13.66 & 0.80 & 6.12 & 18.00 & 2.51 \\
\hline V4 & 229 & 20.00 & 33.06 & 126.00 & 17.89 & 0.60 & 4.87 & 11.00 & 2.35 \\
\hline V5 & 368 & 34.00 & 17.43 & 92.00 & 15.58 & 0.00 & 0.04 & 3.90 & 0.36 \\
\hline
\end{tabular}

Note: $\mathrm{DBH}$, diameter at breast height; TH, tree height; Min., minimum; Max., maximum; SD, standard deviation. 
CBERS) had been used for monitoring the dynamics of riparian vegetation. Summary statistics of measured DBH and TH of $P$. euphratica trees were carried out with SPSS 19.0. The distribution map of trees in different vitalities in the study area was done by ArcGIS 10.0 software based on a GIS database which was established by integrating QuickBird data with terrestrial field survey data. All figures were processed with $\mathrm{R}$ (R Development Core Team, 2013).

\section{Results}

\subsection{Structures of TH and DBH of trees in different vitalities}

The structures of TH and DBH of trees are closely associated with the forest environmental factors and other man-made and natural disturbances. Distribution of $\mathrm{TH}$ demonstrates the vertical structure of forests, most importantly, the growth conditions of riparian forests. Distribution of tree number in each DBH class directly reflects the regeneration pattern and morphological characteristics of individual tree. It is not only an important indicator of stand structure, but also displays successional trend of forest stands (Wang and Rennolls, 2005). Therefore, it has been the focus of research in the field of forest ecology. We analyzed the distribution patterns of TH and DBH of P. euphratica trees in different vitalities (Fig. 4 and Fig. 5, respec- tively). The overall values of $\mathrm{TH}$ ranged from 0 to $19 \mathrm{~m}$, and the values of DBH were within a range of $0-125$ $\mathrm{cm}$. Trees more than $15 \mathrm{~m}$ in TH and $60 \mathrm{~cm}$ in DBH appeared sporadically. There were irregular distribution patterns of TH among all vitality classes (Fig. 4). The mean THs of trees ranged from 0.04 (V5) to $6.97 \mathrm{~m}$ (V2). The maximum value of TH in V1 was $19 \mathrm{~m}$. There were no significant differences of THs between V2 and V3 (18.00-18.40 m). The distributions of TH in V4 and V5 were more flat with smaller number of large TH classes.

The DBH distributions of P. euphratica trees were characterized by an irregular shape in different vitality classes (Fig. 5). The mean DBHs of P. euphratica trees from V0 to V4 increased from 15.90 to $33.06 \mathrm{~cm}$, but that of trees classified as V5 $(17.43 \mathrm{~cm})$ were exceptional (Table 2). All trees except for V5 were characterized by a greater variation in DBH. The largest trees had a maximum DBH value of $119 \mathrm{~cm}$ in V3 and $126 \mathrm{~cm}$ in V4. According to the method developed by Lu and Yan (1989) for determining the age of $P$. euphratic based on its TH and DBH variables, we found that a very large proportion of trees classified as healthy and good vitalities were mainly composed of relatively young trees. The values of DBH in V0, V1 and V2 were smaller $(\mathrm{DBH} \leq 5 \mathrm{~cm})$. Especially, trees grouped as V0 exhibited more young individuals, indicating that the regeneration process
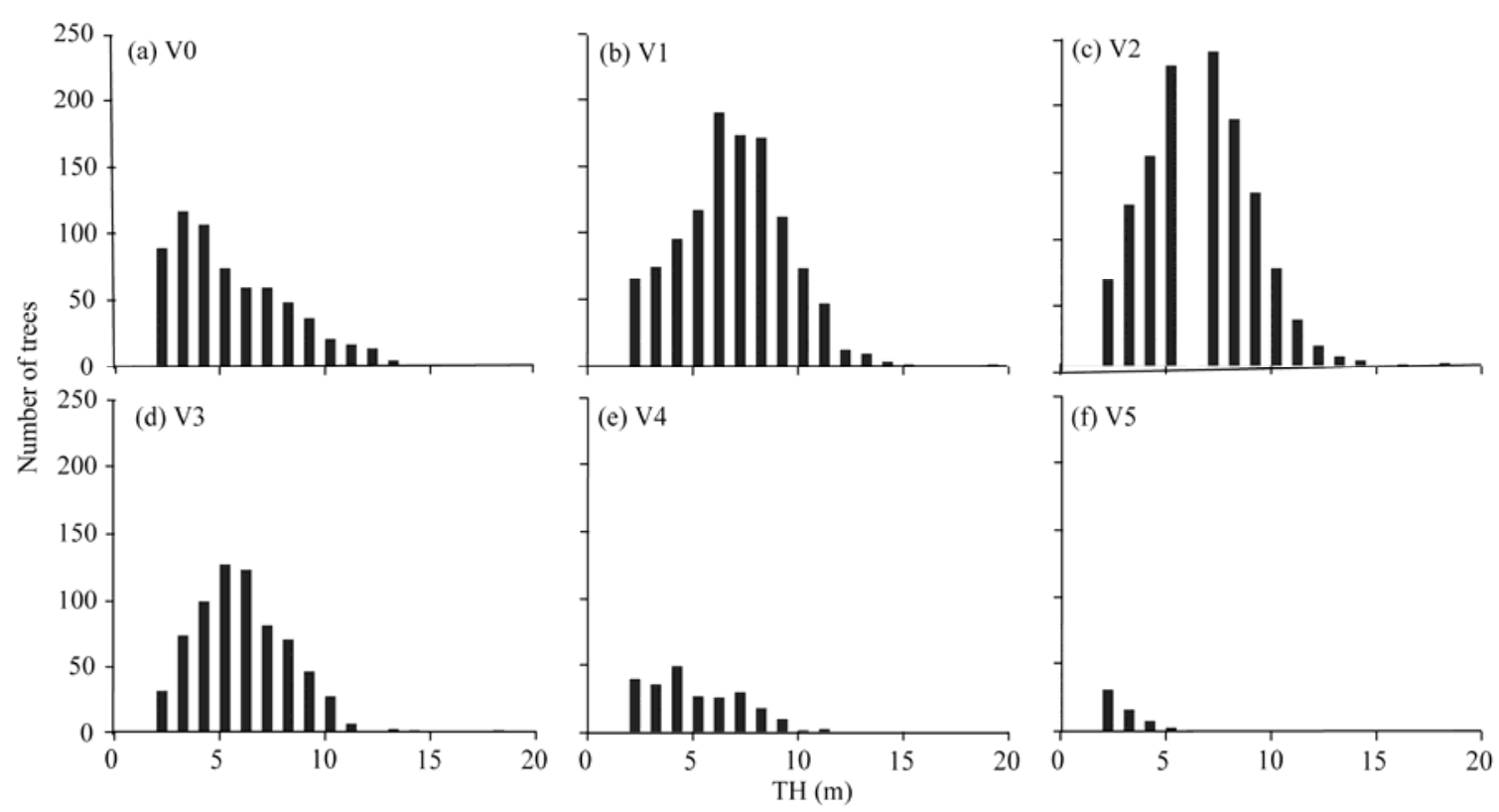

Fig. 4 Structure of tree height (TH) of $P$. euphratica trees in different vitality classes 

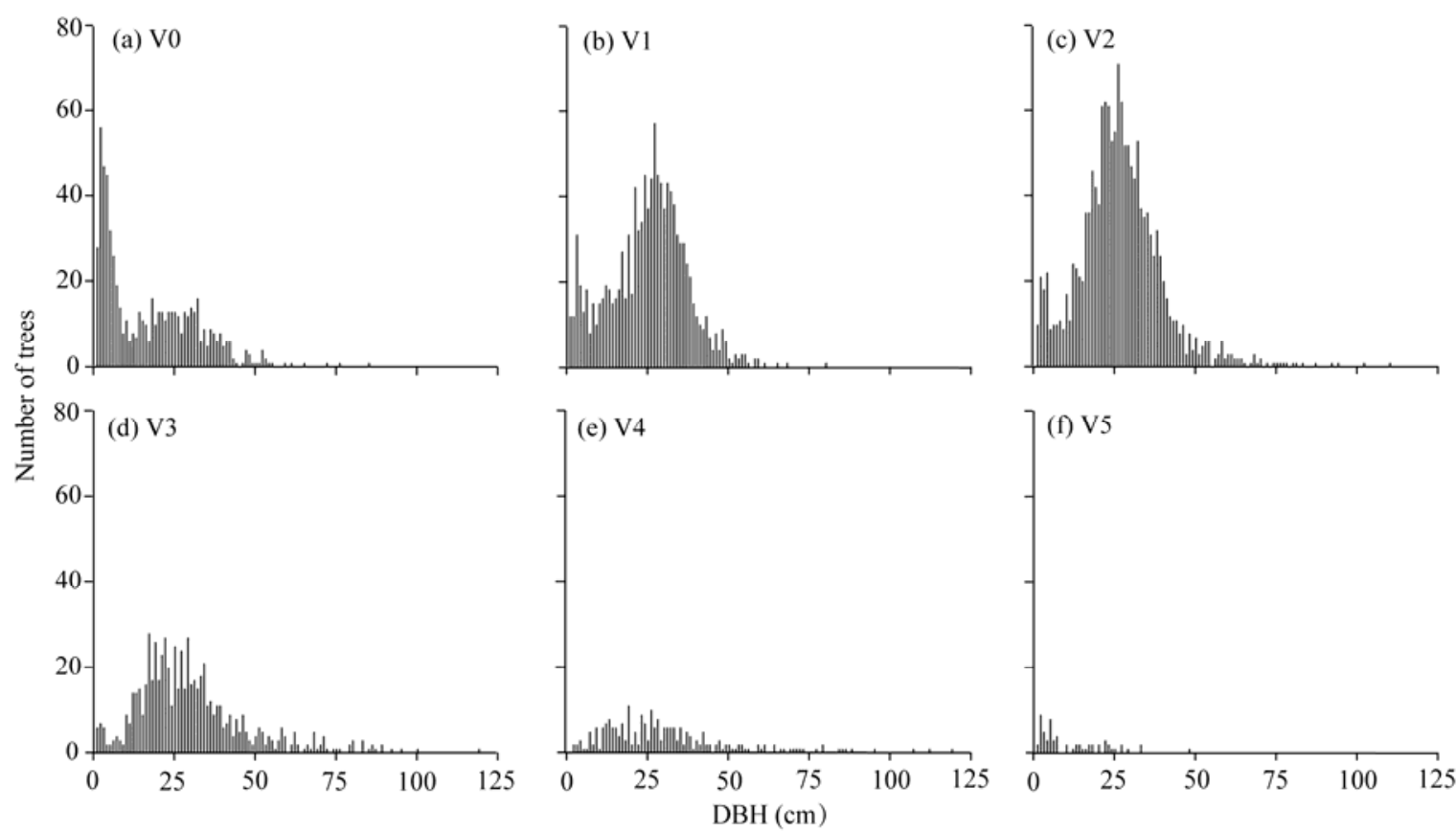

Fig. 5 Structure of diameter at breast height (DBH) of $P$. euphratica trees in different vitality classes

of $P$. euphratica forests under current restoration practices in our study area tends to be still continuing. This showed that $P$. euphratica trees responded to water diversion significantly. It was worth to mention that there were great variations in DBH but not in TH among trees in different vitality classes, suggesting that the DBH growth of $P$. euphratica trees (horizontally) in the lower reaches of the Tarim River seems to be faster than the $\mathrm{TH}$ growth (vertically).

\subsection{Influence of tree vitality on height-diameter relationship}

The TH and DBH of tree are most important variables of forest structure, and are also essential variables in assessing forest condition and modelling stand dynamics (Huang et al., 1992; Zhang, 1997; Maryamgul et al., 2013). Diameter of tree is easily measured compared to height measurement. Either one of these two parameters can be estimated by the height-diameter relationship model (Shawn et al., 2008). Under suitable site conditions, there is a strong biological relationship among different ecological parameters of vegetation. The relationships between $\mathrm{TH}$ and $\mathrm{DBH}$ of $\mathrm{P}$. euphratica trees in different vitality classes are shown in Fig. 6. In this relationship, TH and DBH were taken as the independent and dependent variables, respectively.
We estimated $R^{2}$ obtained from linear regression model for TH and DBH of $P$. euphratica trees at $P<0.001$ significance level. The relationship between TH and DBH in V0 is stronger $\left(R^{2}=0.6942, P<0.001\right)$ than that in other vitality classes. Since majority of vital trees growing at near distance from the river watercourse, $P$. euphratcia has less suffered from water limitation because of favorable groundwater table (Aishan et al., 2013). The height-diameter relationship decreased significantly as the decreasing of tree vitality from V2 to V5. Height-diameter relationships in medium, senesced, dying and dead trees were 0.4247, 0.3489, 0.2352 and 0.1653 , respectively. The main reason for this result could be that the normal growth of trees in these vitality classes is highly limited by groundwater and soil moisture availability (Aishan et al., 2013; Grashey- Jansen et al., 2014).

\section{Discussions}

The structures of TH and DBH of P. euphratica trees showed different distribution patterns. The relationship of height-diameter in $P$. euphratica trees varied remarkably among different vitality classes. Water supply is generally suggested to be responsible for these results. Due to long-term desiccation of river watercourse and continued falling of groundwater 

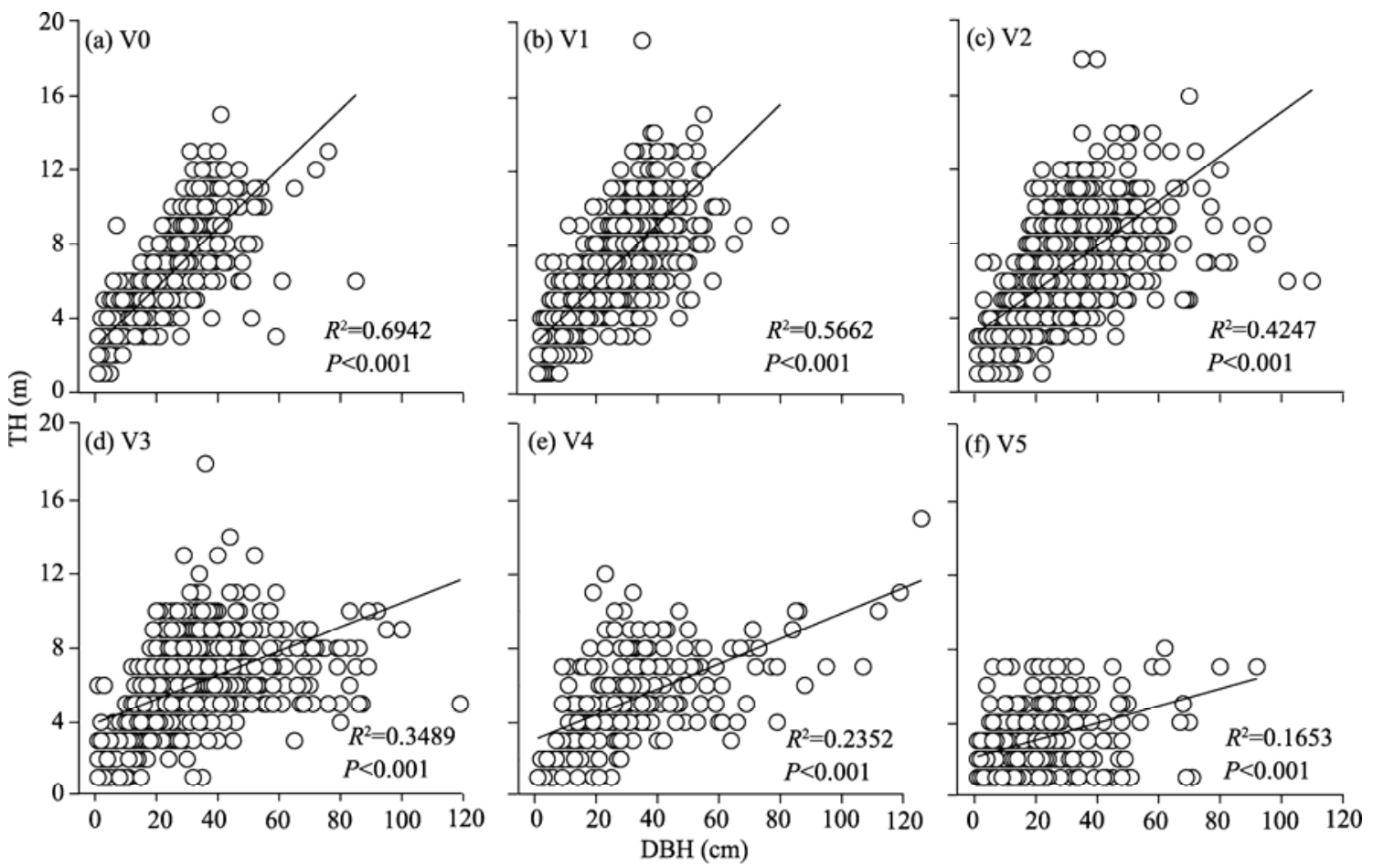

Fig. 6 Correlation between $\mathrm{TH}$ and $\mathrm{DBH}$ of $P$. euphratica trees in different vitality classes

depth (not to meet the needs of riverside vegetation), a majority of riparian forests with weak vitality have lost the ability to renew and regenerate (Westermann et al., 2008; Chen et al., 2010, Aishan et al., 2013; Si et al., 2014). Values of DBH of trees in different vitalities ranged significant, while the distribution pattern of TH was simple. This result indicated that the growth of TH was highly limited compared to the growth of DBH in the lower reaches of the Tarim River. As the implementation of ecological water diversion in the lower reaches of the Tarim River in recent years, the groundwater has obtained a certain degree of supply both quantitatively and qualitatively, and played a positive role in the recovery of $P$. euphratica and other natural plant species (Chen et al., 2006; Xu et al., 2007; Halik et al., 2009). Due to the conflicts between the agricultural irrigation and ecological water demand have become more remarkable, the man-made ecological water conveyance could not be ensured in terms of its flooding time and amount. Therefore, in the vertical direction of watercourses, the river conveyance has been influenced by space constraint to some extent. With increasing distance from the river course, the influencing degree of water conveyance on groundwater recharge decreases cor- respondingly, namely, the more far away from the river course the deeper the groundwater depth (Chen et al., 2006).

At the same time, the vitality of $P$. euphratica forest enervates gradually along the two sides of the river course. The average TH and DBH of P. euphratica trees decreased gradually in the lateral direction to the river course (Fig. 7 and Fig. 8, respectively), since groundwater depth was closely related to the distance from the river course. As P. euphratica tree near to the river course supplied by relatively sufficient water (groundwater and soil moisture), its $\mathrm{TH}$ and DBH were higher than other $P$. euphratica tree which was far away from the river course. A largest number of degraded and stump stand of $P$. euphratica trees can be found at 750 and $1,050 \mathrm{~m}$ away from the river course (near to the desert margin), respectively, indicating that the growth of $P$. euphratica trees is severely limited by water availability. Within 300-m distance from the river course, both structures of $\mathrm{TH}$ and $\mathrm{DBH}$ were relatively diverse. A large variation of $\mathrm{TH}$ and DBH occurred within this corridor. It means that the effects of water diversion on vegetation recovery were significant within this area. Over 300-m distance from the river course, the variations of $\mathrm{TH}$ and $\mathrm{DBH}$ became 
simpler. This result indicated that the factors like tree vitality, distance from the river course and groundwater should be taken into account for designing height-diameter model of degraded riparian forest.

By analyzing the relationships between $\mathrm{TH}$ and DBH of $P$. euphratica in different vitality classes with the distance of trees from the river course, we found that THs of trees in senesced and dying vitality classes were significantly positively correlated with the distance of trees from the river course (Table 3). However, the correlations between THs of trees belonging to other vitality classes and distance of trees from the river course were not significant. In addition to $\mathrm{TH}$ of $P$. euphratica in dead vitality class, the correlation between $\mathrm{TH}$ and distance of trees from the river course increased correspondingly with the decreasing of tree vitality. This showed that the distance of trees from the river course was a major factor determining the vitality of $P$. euphratica trees. A majority of healthy trees were located within the near river course where moisture conditions were sufficient. Therefore, the distance of trees from the river course does play a

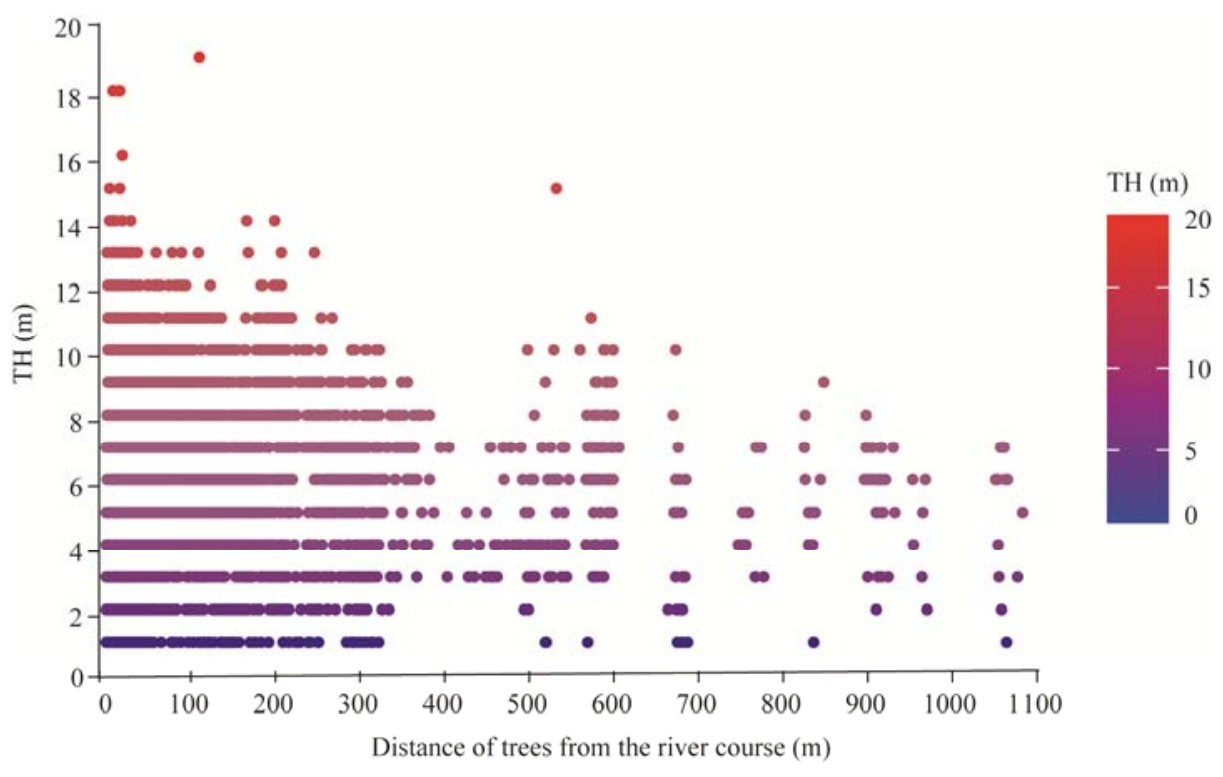

Fig. 7 TH distribution of $P$. euphratica trees at different distances from the river course

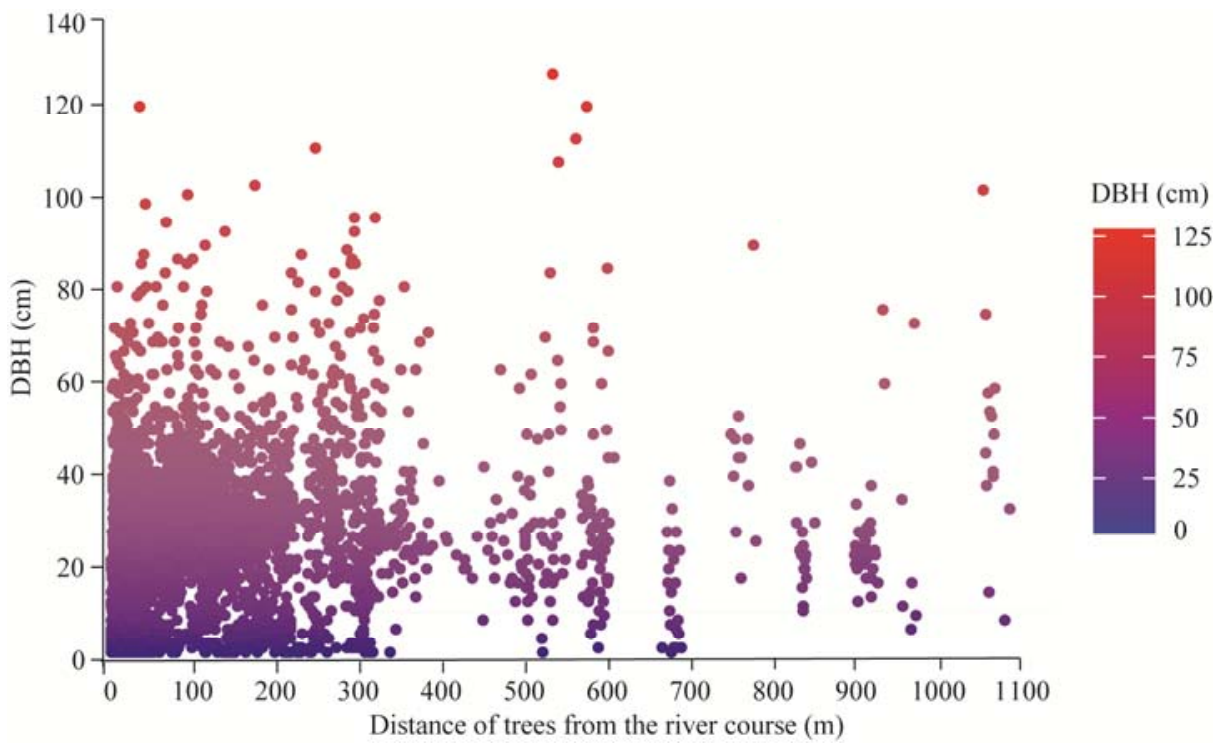

Fig. 8 DBH distribution of $P$. euphratica trees at different distances from the river course 
Table 3 Relationships between TH and DBH of $P$. euphratica trees in different vitality classes with distance of trees from the river course

\begin{tabular}{|c|c|c|c|c|c|c|c|}
\hline \multirow{2}{*}{ Parameter } & \multirow{2}{*}{$\begin{array}{l}\text { Statistic } \\
\text { variable }\end{array}$} & \multicolumn{6}{|c|}{ Vitality class } \\
\hline & & V0 & V1 & V2 & V3 & V4 & V5 \\
\hline \multirow{2}{*}{$\mathrm{TH}$} & $r$ & 0.014 & 0.027 & 0.025 & $0.730^{*}$ & $0.209^{* *}$ & 0.024 \\
\hline & $P$ & 0.692 & 0.346 & 0.317 & 0.048 & 0.001 & 0.609 \\
\hline \multirow{2}{*}{$\mathrm{DBH}$} & $r$ & 0.046 & $1.000^{* *}$ & $0.091^{* *}$ & $0.141^{* *}$ & $0.539^{* *}$ & 0.049 \\
\hline & $P$ & 0.240 & 0.000 & 0.000 & 0.000 & 0.000 & 0.318 \\
\hline
\end{tabular}

Note: $r$ is correlation coefficient; $P$ is significance level. ${ }^{*}$ and ${ }^{* *}$ mean significance at $P<0.05$ and $P<0.01$ (two-tailed) levels, respectively.

key role in determining the tree vitality. Identically, there were significant positive correlations between DBHs of P. euphratica in V1, V2, V3 and V4 with distance of trees from the river course, while DBHs of healthy and dead trees were not correlated with distance of trees to the river course. Possible explanation for this phenomenon is that most of healthy trees are dominantly distributed in the near river course (Aishan et al., 2015). Majority of highly degraded trees may have already lost contact with groundwater so that they cannot be revitalized under current restoration measures.

\section{Conclusion}

The results of this study can be summarized as follows. The TH and DBH structures of $P$. euphratica forests in different vitality classes had irregular distribution patterns. Values of DBH of trees in different vitalities had great variations while the distribution pattern of $\mathrm{TH}$ was simple, indicating that the DBH growth of $P . e u-$ phratica (horizontally) in the lower reaches of the Tarim River seemed to be faster than the TH growth (vertically). Trees classified as healthy, good and medium classes were consisted of large numbers of juvenile trees $(\mathrm{DBH} \leq 5 \mathrm{~cm})$. Especially, healthy trees were composed of relatively young individuals, which suggested that the regeneration process of degraded $P$. euphratica forests by current water diversion projects tends to be still ongoing. Height-diameter relationships of $P$. euphratica trees showed significant variations in different vitality classes. There was a clear trend that the coefficients of height-diameter correlation decreased as the decreasing of tree vitality. All in all, differentiating the degraded $P$. euphratica trees from the healthy trees might be possible by determining the height-diameter correlation coefficient and this coefficient would be a new parameter for detecting the deg- radation level of riparian forests in arid region.

\section{Acknowledgements}

This research was supported by International Science \& Technology Cooperation Program of China (2010DFA92720-12), the National Natural Science Foundation of China (31360200), the German Volkswagen Foundation EcoCAR Project (Az88497) and the German Federal Ministry of Education and Research (BMBF) within the framework of the SuMaRiO Project (01LL0918D). We thank Forestry Department of Qarkilik (Ruoqiang) for their logistic support during our field work in Arghan. The authors would also like to thank the anonymous reviewers for their valuable comments and suggestions to improve the quality of the manuscript.

\section{References}

Aishan T, Halik Ü, Cyffka B, et al. 2013. Monitoring the hydrological and ecological response to water diversion in the lower reaches of the Tarim River, Northwest China. Quatenary International, 311: 155-162.

Aishan T, Halik Ü, Kurban A, et al. 2015. Eco-morphological response of floodplain forests (Populus euphratica Oliv.) to water diversion in the lower Tarim River, northwest China. Environmental Earth Sciences, 73(2): 533-545.

Chen Y N, Zilliacus H, Li W H, et al. 2006. Ground-water level affects plant species diversity along the lower reaches of the Tarim River, Western China. Journal of Arid Environments, 66: 231-246.

Chen Y N, Chen Y P, Xu C C. 2010. Effects of ecological water conveyance on groundwater dynamics and riparian vegetation in the lower reaches of Tarim River, China. Hydrological Process, 24: 170-177.

Chen Y N, Xu C C, Chen Y P, et al. 2013. Progress, challenges and prospects of eco-hydrological studies in the Tarim river basin of Xinjiang, China. Environmental Management, 51(1): 138-153.

Chen Y N, Li W H, Zhou H H, et al. 2014. Analysis of water use strategies of the desert riparian forest plant community in inland rivers of two arid regions in northwestern China. Biogeosciences, 11(10): 14819-14856.

Cyffka B, Rumbaur C, Kuba M, et al. 2013. Sustainable management of river oases along the Tarim River, P.R. China (SuMaRiO) and the ecosystem services approach. Geography, Society, Environment, 6(4): 77-90.

Grashey-Jansen S, Kuba M, Cyffka B, et al. 2014. Spatio-temporal variability of soil water at three seasonal floodplain sites: a case 
study in Tarim Basin, Northwest China. Chinese Geographical Science, 24(6): 647-657.

Halik Ü, Kurban A, Mijit M, et al. 2006. The potential influence of enbankment engineering and ecological water transfer on the riparian vegetation along the middle and lower reaches of the Tarim River. In: Hoppe T, Kleinschmit B, Roberts B, et al. Watershed and Floodplain Management along the Tarim River in 'China's Arid Northwest. Aachen: Shaker, 432.

Halik Ü, Chai Z, Kurban A, et al. 2009. The positive response of some ecological indices of Populus euphratica to the emergency water transfer in the lower reaches of the Tarim River. Journal of Rescources Science, 31: 1309-1314. (in Chinese)

Hao X M, Li W H. 2014. Impacts of ecological water conveyance on groundwater dynamics and vegetation recovery in the lower reaches of the Tarim River in northwest China. Environmental Monitoring and Assessment, 186(11): 7605-7616.

Huang P. 2002. Irrigation-free Vegetation and It's Recovery in Arid Region. Beijing: Science Press, 15-50. (in Chinese)

Huang S M, Stephen J T, Douglas P W. 1992. Comparison of nonlinear height-diameter functions for major Alberta tree species. Canadian Journal of Forest Research, 22(9): 1297-1304.

Kuba M, Aishan T, Cyffka B, et al. 2013. Analysis of connections between soil moisture, groundwater level and vegetation vitality along two transects at the lower reaches of the Tarim River, Northwest China. Geo-Öko, 34(1-2): 103-128.

Lam T Y, Kleinn C, Coenradie B. 2010. Double sampling for stratification for the monitoring of sparse tree populations: the example of Populus euphratica Oliv. forests at the lower reaches of Tarim River, Southern Xinjiang, China. Environmental Monitoring and Assessment, 175(4): 45-61.

Li H K, Fa L. 2011. Height-diameter model for major tree species in China using the classified height method. Scientia Silvae Sinicae, 47(10): 83-90. (in Chinese)

Li L P, Anwar M, Wang X P. 2011. Study on relationship between height and DBH of mountain coniferous forests in Xinjiang. Arid Zone Research, 28(1): 47-53. (in Chinese)

Ling H B, Xu H L, Fu J Y. 2014. Changes in intra-annual runoff and its response to climate change and human activities in the headstream areas of the Tarim River Basin, China. Quaternary International, 336(26): 158-170.

Liu G L, Kurban A, Duan H M, et al. 2014. Desert riparian forest colonization in the lower reaches of Tarim River based on remote sensing analysis. Environmental Earth Sciences, 71(10): 4579-4589.

Lu P, Yan G X. 1989. Forest of Xinjiang. Urumqi: The People's Press of Xinjiang, 226-227. (in Chinese)

Maryamgul A, Kurban A, Halik U, et al. 2013. Study on phenological characters of Populus euphratica Oliv. and its relation with the tree diameter. Vegetos, 26(3): 88-92.

Peng S H, Chen X, Qian J, et al. 2014. Spatial pattern of Populus euphratica forest change as affected by water conveyance in the
Lower Tarim River. Forests, 5(1): 134-152.

R Development Core Team. 2013. R: A Language and Environment for Statistical Computing. Vienna: $\mathrm{R}$ Foundation for Statistical Computing.

Shawn X M, Huang S M, Victor J L, et al. 2008. Wind speed and crown class influence the height-diameter relationship of lodgepole pine: Nonlinear mixed effects modeling. Forest Ecology and Management, 256: 570-577.

Si J H, Feng Q, Cao S H, et al. 2014. Water use sources of desert riparian Populus euphratica forests. Environmental Monitoring and Assessment, 186(9): 5469-5477.

Song Y D, Fan Z L, Lei Z D, et al. 2000. Research on Water Resources and Ecology of the Tarim River, China. Urumqi: The People's Press of Xinjiang, 481. (in Chinese)

Thevs N, Zerbe S, Schnittler M, et al. 2008. Structure, reproduction and flood-induced dynamics of riparian Tugai forests at the Tarim River in Xinjiang, NW China. Forestry, 81(1): 45-57.

Thevs N, Buras A, Zerbe S, et al. 2012. Structure and wood biomass of near-natural floodplain forests along the Central Asian rivers Tarim and Amu Darya. Forestry, 85(2): 193-202.

Wang M, Rennolls K. 2005. Tree diameter distribution modelling: introducing the logit logistic distribution. Canadian Journal of Forest Research, 35(6): 1305-1313.

Wang S C, Li B H, Li H Q. 1996. Euphrates Poplar Forest. Beijing: China Environmental Sciences Press, 22-27. (in Chinese)

Wang X P, Fang J Y, Tang Z Y, et al. 2006. Climatic control of primary forest structure and DBH-height allometry in Northeast China. Forest Ecology and Management, 234: 264-274.

Westermann J, Zerbe S, Eckstein D. 2008. Age structure and growth of degraded Populus euphratica floodplain forests in Northwest China and perspectives for their recovery. Journal of Integrative Plant Biology, 50: 536-546.

Xu H L, Ye M, Li J M. 2007. Changes in groundwater levels and the response of natural vegetation to transfer of water to the lower reaches of the Tarim River. Journal of Environmental Science, 19: 1199-1207.

Yang Y, Yi L. 2014. Spatio-temporal analysis of urbanization and land and water resources efficiency of oasis cities in Tarim River Basin. Journal of Geographical Sciences, 24(3): 509-525.

Ye Z X, Chen Y N, Zhang X. 2014. Dynamics of runoff, river sediments and climate change in the upper reaches of the Tarim River, China. Quaternary International, 336(26): 13-19.

Yukako M, Naoko M, Ken Y. 2008. Stand structure and regeneration of Populus euphratica forest in the lower reaches of the Heihe River, NW China. Landscape and Ecological Engineering, 4: 115-124.

Zhang L. 1997. Cross-validation of non-linear growth functions for modelling tree height-diameter relationships. Annals of Botany, 79: 251-257. 